\title{
On the projective geometry of paths
}

\author{
By J. HaAntJes, University of Edinburgh. \\ (Received 23rd February, 1937. Read 5th March, 1937.)
}

\section{INTRODUCTION.}

An affine connection in an $n$-dimensional manifold $X_{n}$ defines a system of paths, but conversely a connection is not defined uniquely by a system of paths. It was shown by $\mathrm{H}$. Weyl ${ }^{1}$ that any two affine connections whose components are related by an equation of the form

$$
{ }^{\prime} \Gamma_{j i}^{h}=\Gamma_{j i}^{h}+p_{j} A_{i}^{h}+p_{i} A_{j}^{h},
$$

where $A_{i}^{h}$ is the unit affinor', give the same system of paths. In the geometry of a system of paths, a particular parameter on the paths, called the projective normal parameter, plays an important part. This parameter, which is invariant under a transformation of connection (1), was introduced by J. H. C. Whitehead ${ }^{3}$. It can be defined by means of a Schwarzian differential equation and it is determined up to linear fractional transformations ${ }^{4}$. In $\$ 1$ this method is briefly discussed.

In $\S 2$ another method of treating the projective geometry of paths is given, based upon the introduction of homogeneous coordinates in an $n$-dimensional manifold ${ }^{5}$. Instead of one parameter two homogeneous parameters $u^{0}, u^{1}$ are introduced on each path. This leads to a set of coefficients of a projective connection on each path. Then a preferred system of projective parameters is obtained by putting

1 H. Weyl, "Zur Infinitesimalgeometrie: Einordnung der projektiven und der konformen Auffassung," Göttinger Nachrichten (1921), pp. 99-112.

2 In this paper the term " affinor" is used instead of "tensor."

3 J. H. C. Whitehead, "The representation of projective spaces," Ann. of Math., 32 (1931), pp. 327-360.

4 L. Berwald, "On the projective geometry of paths," Ann. of Math., 37 (1936), pp. 879-898.

${ }^{5} \mathrm{D}$. van Dantzig, "Theorie des projektiven Zusammenhangs $n$-dimensionaler Räume," Math. Annalen 106 (1932), pp. 400-454. See also J. A. Schouten and J. Haantjes, "Zur allgemeinen projektiven Differentialgeometrie," Compositio Math. 3 (1935), pp. 1-51. This paper is referred to as A. P. D. 
these coefficients equal to zero. Such a preferred system is determined up to linear homogeneous transformations with constant coefficients. Hence the ratio $p=u^{1} / u^{0}$ is a non-homogeneous parameter, which is defined up to linear fractional transformations. In $\S 3$ it is shown that the parameter $p$ is a projective normal parameter.

\section{$\S 1$. Paths and affine connections.}

1. Paths in $L_{n}$.

We consider an $n$-dimensional manifold $L_{n}$, in which a symmetrical affine connection $\Gamma_{j i}^{h}$ is given. The coordinates of a point are denoted by $\xi^{h}(h, \ldots, m=1, \ldots, n)$. A coordinate transformation is given by a set of $n$ analytic functions

$$
\xi^{h^{\prime}}=\xi^{h^{\prime}}\left(\xi^{1}, \ldots, \xi^{n}\right),
$$

whose functional determinant is different from zero for all points under consideration.

By a path we mean a curve $\xi^{h}=\xi^{h}(t)$, where $\xi^{h}(t)$ is a solution of the following system of differential equations

$$
\frac{d^{2} \xi^{h}}{d t^{2}}+\Gamma_{j i}^{h} \frac{d \xi^{j}}{d t} \frac{d \xi^{i}}{d t}=\beta \frac{d \xi^{h}}{d t},
$$

$\beta$ being a function of $t$. Thus the paths are autoparallel curves. It is possible to introduce a new parameter $s=s(t)$ on each curve, such that the differential equations take the form

$$
\frac{d^{2} \xi^{h}}{d s^{2}}+\Gamma_{j i}^{h} \frac{d \xi^{j}}{d s} \frac{d \xi^{i}}{d s}=0
$$

The parameter $s$ is called an affine parameter of the system of paths. On each path it is determined up to an arbitrary linear transformation $s^{\prime}=a s+b, a$ and $b$ being arbitrary constants.

2. Projective transformations of an affine connection.

An affine connection defines a system of paths, but a system of paths (1.2) does not define a symmetrical connection uniquely. For the equations (1.2) remain unaltered if we put in the place of $\Gamma_{j i}^{h}$ the functions

$$
' \Gamma_{j i}^{h}=\Gamma_{j i}^{h}+p_{j} A_{i}^{h}+p_{i} A_{j}^{h},
$$

where $p_{i}$ is an arbitrary covariant vector and $A_{j}^{h}$ denotes the unit affinor. Such a transformation of connection is called a projective transformation of the affine connection ${ }^{1}$. In general it changes the parameter $s$.

1 B. Weyl, l. c. 
The object with components

$$
\Pi_{j i}^{h}=\Gamma_{j i}^{h}-\frac{1}{n+1}\left(A_{j}^{h} \Gamma_{i l}^{l}+A_{i}^{h} \Gamma_{j l}^{l}\right)
$$

is unaltered by projective transformations of connection. These $\Pi_{j i}^{h}$, which satisfy the identity $\Pi_{j h}^{h}=0$ are called the Thomas parameters ${ }^{1}$.

By replacing $\Gamma_{j i}^{h}$ in (1.2) by $\Pi_{j i}^{h}$ we get the same set of curves. The parameter $s$ corresponding to $\Pi_{j i}^{h}$, that is the parameter $s$ for which the differential equations have the form

$$
\frac{d^{2} \xi^{h}}{d s^{2}}+\Pi_{j i}^{h} \frac{d \xi^{j}}{d s} \frac{d \xi^{i}}{d s}=0
$$

is called the projective parameter of $T$. Y. Thomas. It does not change under projective transformations of connection. But since the $\Pi_{j i}^{h}$ are not transformed under a transformation of coordinates like the components of an affine connection, this projective parameter is not a scalar.

3. The projective normal parameter.

The curvature affinor of the affine connection $\Gamma_{j i}^{h}$ is defined by

$$
R_{\dot{k}_{j i}{ }^{h}}^{h}=2 \partial_{[k} \Gamma_{j] i}^{h}+2 \Gamma_{\lceil k|l|}^{h} \Gamma_{j] i}^{l}, \quad\left(\partial_{j}=\frac{\partial}{\partial \xi^{j}}\right)
$$

where the square brackets mean alternation with respect to the indices $k$ and $j$ (for example, $2 \partial_{[k} w_{i]}=\partial_{k} w_{i}-\partial_{i} w_{k}$ ). Contraction gives the affinor

$$
R_{j i}=R_{\not{h j i}}^{h} \text {. }
$$

A projective normal parameter $\pi$ on the paths (1.2) is now defined by means of a differential equation of the form

$$
\{\pi, s\}=\frac{2}{n-1} R_{h i} \frac{d \xi^{h}}{d s} \frac{d \xi^{i}}{d s},
$$

where $s$ is an affine parameter belonging to the connection $\Gamma_{j i}^{h}$ and $\{\pi, s\}$ stands for the Schwarzian derivative

$$
\{\pi, s\}=\frac{\frac{d^{3} \pi}{d s^{3}}}{\frac{d \pi}{d s}}-\frac{3}{2}\left(\frac{\frac{d^{2} \pi}{d s^{2}}}{\frac{d \pi}{d s}}\right)^{2}
$$

${ }^{3}$ T. Y. Thomas, "On the projective and equiprojective geometry of paths," Proc. Nat. Acad. Sci., U.S.A. 11 (1925), pp. 199-203; "A projective theory of affinely connected manifolds," Math. Zeitschrift 25 (1926), pp. 723-733. 
By (1.9) $\pi$ is defined as function of $s$ up to linear fractional transformations. It can be proved that a projective normal parameter of a system of paths has the following properties ${ }^{1}$ :

(a) It is not altered by transformations of coordinates, which means that $\pi$ is a scalar.

(b) It is not altered by projective transformations of the connection.

If this parameter $\pi(s)$ is introduced in the differential equations (1.3) these equations take the form ${ }^{2}$

$$
\frac{d^{2} \xi^{h}}{d \pi^{2}}+\Gamma_{j i}^{h} \frac{d \xi^{j}}{d \pi} \frac{d \xi^{i}}{d \pi}+2 \alpha \frac{d \xi^{h}}{d \pi}=0
$$

where $a$ satisfies the equation

$$
2 a\left(\frac{d \pi}{d s}\right)^{2}=\frac{d^{2} \pi}{d s^{2}}
$$

From this equation and $(1 \cdot 9)(1 \cdot 10)$ it follows by differentiation

$$
\frac{d a}{d \pi}+a^{2}-\frac{1}{n-1} R_{h i} \frac{d \xi^{h}}{d \pi} \frac{d \xi^{i}}{d \pi}=0 .
$$

It can be shown that conversely the equations (1.11) and (1.13) determine a projective normal parameter $\pi$.

§2. Paths and projective connections.

1. Paths in $H_{n}$.

We introduce in the $n$-dimensional manifold homogeneous coordinates $x^{\kappa},(\kappa, \ldots, \tau=0,1, \ldots, n)$, and subject these coordinates to the set of transformations

$$
x^{\kappa^{\prime}}=x^{\kappa^{\prime}}\left(x^{0}, \ldots, x^{n}\right),
$$

where the $x^{x^{\prime}}$ are homogeneous analytic functions of the first degree in the $x^{\kappa}$, such that the functional determinant is different from zero for all points under consideration. Such an $n$-dimensional manifold with homogeneous coordinates is called a generalized projective space ${ }^{3}$ and is denoted by $H_{n}$. A particular property of an $H_{n}$ is that the coordinates $x^{\kappa}$ of a point transform like the components of a projective contravariant vector, for we have from (2.1) according to Euler's condition of homogeneity

1 J. H. C. Whitehead, l. c. ; L. Berwald, l.c., p. 882.

J. H. C. Whitehead, l.c., p. 338 ; L. Berwald, l. c., p. 884.

3 D. van Dantzig, l. c. 


$$
x^{\kappa^{\prime}}=x^{\kappa} \partial_{\kappa} x^{\kappa^{\prime}}=\mathcal{A}_{\kappa}^{\kappa \prime} x^{\kappa}, \partial_{\mu}=\frac{\partial}{\partial x^{\mu}}, \mathscr{A}_{\kappa}^{\kappa \prime}=\partial_{\kappa} x^{\kappa^{\prime}} .
$$

A covariant derivative in the $H_{n}$ is given by $(n+1)^{3}$ functions $\Pi_{\mu \lambda}^{\kappa}$ called the coefficients of the projective connection. These coefficients are homogeneous functions of $x^{k}$ of degree -1 . From the transforma. tion formula for the coefficients $\Pi_{\mu \lambda}^{\kappa}$

it follows that the

$$
\begin{aligned}
& \Pi_{\mu^{\prime} \lambda^{\prime}}^{\alpha^{\prime}}=\mathscr{A}_{\kappa}^{\alpha^{\prime} \mu} \mu_{\mu^{\prime}}^{\lambda}, \Pi_{\mu_{\lambda}}^{\kappa}+\mathscr{A}_{\kappa}^{\alpha \prime} \partial_{\mu^{\prime}} \mathscr{A}_{\lambda^{\prime}}^{\kappa} ; \\
& \mathscr{A}_{\kappa}^{\alpha \prime} \mu_{\mu^{\prime} \lambda^{\prime}}=\mathscr{A}_{\kappa}^{\alpha^{\prime}} \mathscr{A}_{\mu^{\prime}}^{\mu} \mathscr{A}_{\lambda^{\prime}}^{\lambda} ; \mathscr{A}_{\lambda^{\prime}}^{\lambda}=\partial_{\lambda^{\prime}} x^{\lambda} ;
\end{aligned}
$$

$$
\Pi_{\mu \lambda}^{\kappa} x^{\mu}
$$

transform like the components of a projective affinor. Hereafter homogeneous projective affinors will be called projectors.

In an $H_{n}$ the equations $x^{\kappa}=f^{\kappa}(t)$ and $x^{\kappa}=\rho(t) f^{\kappa}(t)$ define the same curve. From this it follows that the differentials $d x^{\kappa}$ define the same direction as $\rho d x^{\kappa}+x^{\kappa} d \rho$. In other words, the vectors

$$
\frac{d x^{\kappa}}{d t} \text { and } \rho \frac{d x^{\kappa}}{d t}+\frac{d \rho}{d t} x^{\kappa}
$$

define the same direction. We restrict ourselves to symmetrical connections with the property that the projector (2.4) is zero, hence

$$
\Pi_{\mu \lambda}^{\kappa}=\Pi_{\lambda \mu}^{\kappa}, \quad \Pi_{\mu \lambda}^{\kappa} x^{\mu}=0 .
$$

A result of the hypothesis $\Pi_{\mu_{\lambda}}^{\kappa} x^{\mu}=0$ is that there exists a displacement for a direction in its own direction. For if the homogeneous vector $v^{\text {k }}$ satisfies the relation

$$
d x^{\mu} \nabla_{\mu} v^{\kappa}:: v^{\kappa},
$$

then it satisfies the relation, which is obtained from (2.6) by putting $\rho d x^{\mu}+x^{\mu} d \rho$ instead of $d x^{\mu}$. Thus in this case a path can be defined as an autoparallel curve. In a more general $H_{n}$ autoparallel curves need not exist.

If the curve $x^{\kappa}=x^{\kappa}(t)$ is a path (autoparallel curve), then the vector

$$
\frac{d x^{\mu}}{d t} \nabla_{\mu} \frac{d x^{\kappa}}{d t}
$$

has the same direction as $\frac{d x^{\kappa}}{d t}$. Therefore, we find for the differential equation of the paths

$$
\frac{d x^{\mu}}{d t} \nabla_{\mu} \frac{d x^{\kappa}}{d t}=\frac{d^{2} x^{\kappa}}{d t^{2}}+\Pi_{\mu \lambda}^{\kappa} \frac{d x^{\mu}}{d t} \frac{d x^{\lambda}}{d t}=\alpha x^{\kappa}+\beta \frac{d x^{\kappa}}{d t} .
$$


In these equations $a$ and $\beta$ depend on $t$. The equations (2.7) define $\infty^{2(n-2)}$ paths, such that through each point in a certain region of $H_{n}$ there is a unique path in each direction.

The projector of curvature is defined by

$$
N_{i \mu \dot{\lambda}}{ }^{\kappa}=2 \partial_{[\nu} \Pi_{\mu] \lambda}^{\kappa}+2 \Pi_{[\nu|\rho|}^{\kappa} \Pi_{\mu] \lambda}^{\rho} .
$$

Transvection with $x^{\nu}$ gives

$$
N_{\nu \mu \dot{\lambda}}{ }^{\kappa} x^{\nu}=x^{\nu} \partial_{\nu} \Pi_{\mu \lambda}^{\kappa}-x^{\nu} \partial_{\mu} \Pi_{\nu \lambda}^{\kappa}=-\Pi_{\mu \lambda}^{\kappa}+\Pi_{\mu \lambda}^{\kappa}=0 .
$$

2. The transformations of the projective connection, which leave the system of paths invariant.

A projective connection, satisfying (2.5), defines a system of paths, but conversely the system of paths does not define uniquely a symmetrical projective connection. Indeed, the transformation of connection ${ }^{1}$

$$
{ }^{\prime} \Pi_{\mu \lambda}^{\kappa}=\Pi_{\mu \lambda}^{\kappa}+Z_{\mu \lambda} x^{\kappa}+z_{\mu} \mathscr{A}_{\lambda}^{\kappa}+z_{\lambda} \mathscr{A}_{\mu}^{\kappa},
$$

where $Z_{\mu \lambda}$ and $z_{\mu}$ are arbitrary projectors, homogeneous of degree -2 and -1 respectively and $\mathscr{A}_{\lambda}^{*}$ denotes the unit projector, leaves the system of paths, defined by (2.7), invariant. The restriction (2.5) gives

$$
Z_{\mu \lambda}=Z_{\lambda \mu}, \quad Z_{\mu \lambda} x^{\mu}+z_{\lambda}=0, \quad z_{\mu} x^{\mu}=0 .
$$

The projectors $Z_{\mu \lambda}$ and $z_{\lambda}$ can be chosen in such a way that the contracted projector of curvature ' $N_{\mu \lambda}={ }^{\prime} N_{\kappa} \mu_{\mu}{ }^{*}$ of the new connection $' \Pi_{\mu_{\lambda}}^{\kappa}$ vanishes. On calculation we find ${ }^{2}$

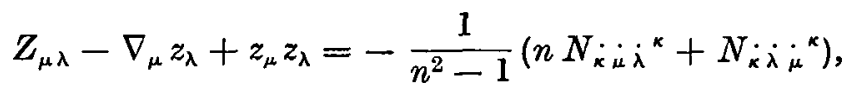

so that

$$
(n+1) \nabla_{[\mu} z_{\lambda]}=N_{\dot{\kappa}[\dot{\mu} \dot{\lambda}]}{ }^{\kappa}=N_{[\mu \lambda]} .
$$

This equation is easily shown to be integrable by use of Bianchi's identity, and determines $z_{\lambda}$ but for a gradient vector. From (2.12) and (2.13) we obtain the following theorem.

A system of paths (2.7) determines a symmetrical projective connection with

$$
\Pi_{\mu \lambda}^{\kappa} x^{\mu}=0, \quad N_{\mu \lambda}=0
$$

\footnotetext{
${ }^{2}$ A. P. D., p. 32.

A P. D., p. 33.
} 
up to a transformation of the form (2.10), where $Z_{\mu \lambda}$ and $z_{\lambda}$ satisfy, besides (2.11) the following equations

$$
\begin{aligned}
Z_{\mu \lambda}-\nabla_{\mu} z_{\lambda}+z_{\mu} z_{\lambda} & =0 \\
\nabla_{[\mu} z_{\lambda]} & =0 .
\end{aligned}
$$

The equation $(2.15 \mathrm{~b})$ means that $z_{\lambda}$ is a gradient vector, $z_{\lambda}=\partial_{\lambda} z$.

3. The projective parameter p.

Let us now introduce two homogeneous parameters on the paths. A path $x^{\kappa}=x^{\kappa}(t)$ may also be given by the equations

$$
x^{\kappa}=x^{\kappa}\left(u^{0}, u^{1}\right)=x^{\kappa}\left(u^{a}\right), \quad(a, \ldots, g=0,1)
$$

where the $x^{\kappa}\left(u^{0}, u^{1}\right)$ are homogeneous functions of degree $1^{1}$. Then $u^{a}$ and $\sigma u^{a}$ determine the same point on the curve. A transformation of homogeneous parameters is given by a set of functions

$$
u^{a^{\prime}}=u^{a^{\prime}}\left(u^{0}, u^{1}\right)
$$

homogeneous of degree 1. From (2.16) and (2.17) it follows from Euler's condition of homogeneity

$$
\begin{aligned}
& x^{\kappa}=B_{a}^{\kappa} u^{a}, \quad B_{a}^{\kappa}=\partial_{a} x^{\kappa}, \quad \partial_{a}=\frac{\partial}{\partial u^{a}} \\
& u^{a \prime}=B_{a}^{a \prime} u^{a}, \quad E_{a}^{a \prime}=\partial_{a} u^{a^{\prime}} .
\end{aligned}
$$

The vectors $B_{0}^{\kappa}$ and $B_{1}^{\kappa}$ have the same direction as $\frac{d x^{\kappa}}{d t}$, from which it follows that both $B_{0}^{\kappa}$ and $B_{1}^{\kappa}$ can be expressed linearly in terms of $x^{\kappa}$ and $\frac{d x^{\kappa}}{d t}$

$$
B_{a}^{\kappa}=p_{a} \frac{d x^{\kappa}}{d t}+q_{a} x^{\kappa}
$$

These equations may be solved for $x^{\kappa}$ and $\frac{d x^{\kappa}}{d t}$, giving (c.f. 2.18),

$$
\frac{d x^{\kappa}}{d t}=r^{a} B_{a}^{\kappa}, \quad x^{\kappa}=u^{a} B_{a}^{\kappa}
$$

From (2.20) it follows by covariant differentiation, in consequence of (2.7), that $B_{c}^{\mu} \nabla_{\mu} B_{a}^{\kappa}$ is a linear expression in $x^{\kappa}$ and $\frac{d x^{\kappa}}{d t}$, and therefore, by (2.21), a linear expression in $B_{0}^{x}$ and $B_{1}^{x}$. Hence the differential equations of the paths take the form

$$
B_{c}^{\mu} \nabla_{\mu} B_{b}^{\kappa}=\partial_{c} B_{b}^{\kappa}+\Pi_{\mu \lambda}^{\kappa} B_{c b}^{\mu \lambda}=\Gamma_{c b}^{a} B_{a}^{\kappa}, \quad B_{c b}^{\mu \lambda}=E_{c}^{\mu} B_{b}^{\lambda}
$$

1 The equations are obtainable in this form by putting $t=u^{1} / u^{0}$ in $x^{\star}=x^{\kappa}(t)$ and multiplying by an arbitrary homogeneous function of degree 1 . 
the $\Pi_{\mu \lambda}{ }_{\mu}$ being the coefficients of one of the projective connections, determined by the system of paths, which satisfy the equations (2.14). From (2.22) it follows that the functions $\Gamma_{c b}^{a}$ are homogeneous in $u^{a}$ of degree -1 . When we transform the parameters $u^{a}$ by any transformation (2.17) the coefficients $\Gamma_{c b}^{a}$ transform according to the equations

$$
\Gamma_{c^{\prime} b^{\prime}}^{a^{\prime}}=B_{a c^{\prime} b^{\prime}}^{a^{\prime} c^{\prime}} \Gamma_{c b}^{a}+B_{a}^{a^{\prime} \partial_{c^{\prime}}} B_{b^{\prime}}^{a} ; \quad\left(B_{b^{\prime}}^{a}=\partial_{b^{\prime}} u^{a}\right) .
$$

The $\Gamma_{c b}^{a}$ transform therefore like the coefficients of a projective connection in an $H_{1}$. Moreover we have from (2.22)

$$
\Gamma_{c b}^{a} u^{c}=0 ; \quad \Gamma_{c b}^{a}=\Gamma_{b c}^{a} .
$$

It is well known that a necessary and sufficient condition for the existence of a system of parameters $u^{a}$, such that all of the $\Gamma_{c b}^{a}$ are zero, is that

$$
M_{\dot{d} \dot{c} \dot{b}}{ }^{a}=2 \partial_{[d} \Gamma_{c] b}^{a}+2 \Gamma_{[d|e|}^{a} \Gamma_{c] b}^{e}
$$

be zero. The transvection of $M_{\dot{a} \dot{c} \dot{b}}^{a}$ and $u^{d}$ is (c.f. (2.9))

$$
u^{d} M_{\dot{d} \dot{c} \dot{b}}{ }^{a}=u^{d} \partial_{d} \Gamma_{a b}^{a}-u^{d} \partial_{c} \Gamma_{d b}^{a}=0 .
$$

The quantity $M_{\dot{d} \dot{c} \dot{b}}{ }^{a}$ is skew symmetrical in the indices $d$ and $c$. Therefore, the rank of $M_{\dot{d} \cdot \dot{c} b}{ }^{a}$ with respect to the indices $d$ and $c$ must be either 2 or 0 . The equations (2.26) express that the rank is less than 2 , hence the rank is 0 . Thus we have

$$
M_{\dot{a} \dot{c} \dot{b}}{ }^{a}=0 \text {. }
$$

This means that there exists a system of parameters $u^{a}$ for which $\Gamma_{c b}^{a}=0$ and from the transformation (2.23) of $\Gamma_{c b}^{a}$ it follows that this system is determined up to linear homogeneous transformations with constant coefficients. The non-homogeneous parameter

$$
p=\frac{u^{1}}{u^{0}}
$$

is then determined up to linear fractional transformations. We call this parameter a projective parameter. In $\S 3$ it is proved that $p$ is a projective normal parameter.

We have to prove first that the parameter $p$ is unaltered by a transformation of connection (2.10), where $Z_{\mu \lambda}$ and $z_{\lambda}$ satisfy the conditions (2.11) and (2.15). From (2.15) we have

$$
z_{\mu}=\partial_{\mu} z, Z_{\mu \lambda}=\nabla_{\mu} z_{\lambda}-z_{\mu} z_{\lambda}=\bar{\nabla}_{\mu} \nabla_{\lambda} z-\left(\dot{\partial}_{\mu} z\right)\left(\partial_{\lambda} z\right)
$$

and the function $z$ is homogeneous of degree zero. By (2.22) such a 
transformation of connection causes the following transformation of $\Gamma_{c b}^{a}$

where

$$
\begin{aligned}
{ }^{\prime} \Gamma_{c b}^{a} & =\Gamma_{c b}^{a}+Z_{\mu \lambda} B_{c b}^{\mu \lambda} u^{a}+z_{\mu} B_{c}^{\mu} B_{b}^{a}+z_{\mu} B_{b}^{\mu} B_{c}^{a} \\
& =\Gamma_{c b}^{a}+Z_{c b} u^{a}+z_{c} B_{b}^{a}+z_{b} B_{c}^{a}, \quad B_{b}^{a}\left\{\begin{array}{l}
=1, a=b \\
=0, a \neq b
\end{array}\right.
\end{aligned}
$$

$$
\begin{gathered}
z_{c}=B_{c}^{\mu} \partial_{\mu} z=\partial_{c} z \\
Z_{c b}=B_{c b}^{\mu \lambda}\left(\partial_{\mu} \partial_{\lambda} z-\Pi_{\mu \lambda}^{\kappa} \partial_{\kappa} z\right)-z_{c} z_{b}=\partial_{c} z_{b}-\Gamma_{c b}^{a} z_{a}-z_{c} z_{b} .
\end{gathered}
$$

For a preferred system of parameters $\left(\Gamma_{c b}^{a}=0\right)$ we have therefore

$$
{ }^{\prime} \Gamma_{c b}^{a}=\left(\partial_{c} z_{b}-z_{c} z_{b}\right) u^{a}+z_{c} B_{b}^{a}+z_{b} B_{c}^{a} .
$$

The equations (2.24) hold also for ' $\Gamma_{c b}^{a}$; from which it follows; as we have seen above, that the projector of curvature ' $M_{\dot{d} \dot{c} \dot{b}}{ }^{a}$ belonging to ' $\Gamma_{c b}^{a}$ vanishes. There exist, therefore, systems of parameters $u^{a \prime}$ for which ${ }^{\prime} \Gamma_{c^{\prime} b^{\prime}}^{a \prime}=0$. We shall now show that one of these systems of parameters can be obtained by a transformation of parameters of the following form

$$
\begin{aligned}
& u^{1^{\prime}}=\rho\left(u^{a}\right) u^{1} \\
& u^{0 \prime}=\rho\left(u^{a}\right) u^{0},
\end{aligned}
$$

where $\rho\left(u^{a}\right)$ is a homogeneous function of degree zero. From (2.33) it follows

$$
\begin{aligned}
& B_{a}^{a \prime} \stackrel{*}{=} \rho \delta_{b}^{a^{\prime}}\left(B_{a}^{b}+u^{b} \partial_{a} \log \rho\right) \\
& B_{a^{\prime}}^{a} \stackrel{*}{=} \rho^{-1} \delta_{a^{\prime}}^{b}\left(B_{b}^{a}-u^{a} \partial_{b} \log \rho\right)
\end{aligned}
$$

where the $\delta_{b}^{a \prime}$ denote the generalized Kronecker symbols ${ }^{1}$. Substitution in the transformation formula (2.23) for ' $\Gamma_{c b}^{a}$ gives

$$
\begin{aligned}
\Gamma_{c^{\prime} b^{\prime}}^{a^{\prime}} \stackrel{*}{=} & \rho^{-1} \delta_{a}^{a^{\prime}} \delta_{c^{\prime}}^{c} \delta_{b^{\prime}}^{b}\left[{ }^{\prime} \Gamma_{c b}^{a}+\left\{-\partial_{c} \partial_{b} \log \rho+{ }^{\prime} \Gamma_{c b}^{e} \partial_{e} \log \rho\right.\right. \\
& \left.\left.-\left(\partial_{c} \log \rho\right)\left(\partial_{b} \log \rho\right)\right\} u^{a}-\partial_{b} \log \rho B_{c}^{a}-\partial_{c} \log \rho B_{b}^{a}\right] .
\end{aligned}
$$

By substituting the expression (2.32) for ' $\Gamma_{c b}^{a}$, we get

$$
\begin{array}{r}
\prime \Gamma_{c^{\prime} b^{\prime}}^{a \prime} \stackrel{*}{=} \rho^{-1} \delta_{a}^{a \prime} \delta_{c^{\prime}}^{c} \delta_{b}^{b}\left[\left\{\partial_{c} z_{b}-z_{c} z_{b}-\partial^{c} \partial_{b} \log \rho-\left(\partial_{c} \log \rho\right)\left(\partial_{b} \log \rho\right)\right.\right. \\
\left.\left.\quad+z_{c} \partial_{b} \log \rho+z_{b} \partial_{c} \log \rho\right\} u^{a}+\left(z_{b}-\partial_{b}^{\prime} \log \rho\right) B_{c}^{a}+\left(z_{c}-\partial_{c} \log \rho\right) B_{b}^{a}\right]
\end{array}
$$

and from this equation we see that we get

$$
\Gamma_{c^{\prime} b^{\prime}}^{a^{\prime}} \stackrel{*}{=} 0
$$

by putting

$$
\log \rho=z, \quad \partial_{c} \log \rho=\partial_{c} z=z_{c}
$$

1 The sign $\stackrel{*}{=}$ means that the equation holds with respect to the coordinate system or systems used in the. equation itself; it need not hold with respect to another system. 
Thus it is possible to get a preferred system of homogeneous parameters $u^{a^{\prime}}$ belonging to ' $\Gamma_{c b}^{a}$ from a preferred system of parameters belonging to $\Gamma_{c b}^{a}$ by a transformation of the form (2.33). From (2.28) and $(2.33)$ it follows that

$$
\frac{u^{1^{\prime}}}{u^{0^{\prime}}}=\frac{u^{1}}{u^{0}}=p
$$

Hence, the projective parameter $p$ remains unaltered under a transformation (2.10) of the projective connection. It is, therefore, determined by the system of paths up to linear fractional transformations.

\section{§3. THE TWO PARAMETERS $p$ AND $\pi$.}

1. Introduction of non-homogeneous coordinates in $H_{n}$.

In this paragraph we shall prove that the projective parameters $p$ and $\pi$ are "identical," in other words that $p$ is a projective normal parameter.

In order to compare the parameters $p$ and $\pi$, we have to introduce ${ }^{1}$ non-homogeneous coordinates in $H_{n}$. A system of nonhomogeneous coordinates in $H_{n}$ is given by a set of $n$ functions

$$
\xi^{h}=\xi^{h}\left(x^{0}, \ldots, x^{n}\right),
$$

homogeneous of degree zero, whose functional matrix

$$
\left\|\varepsilon_{\lambda}^{h}\right\|, \text { where } \varepsilon_{\lambda}^{h}=\partial_{\lambda} \xi^{h}
$$

has rank $n$. From (3.1) it follows by Euler's condition of homogeneity that

$$
x^{\mu} \varepsilon_{\mu}^{h}=0 .
$$

Moreover we introduce a projective covariant vector field $q_{\lambda}$ of degree -1 , such that

$$
q_{\lambda} x^{\lambda}=1
$$

But for this equation, $q_{\lambda}$ may be chosen at will. This vector $q_{\lambda}$ enables us to define the inverse of $\varepsilon_{\lambda}^{h}$. We define the quantity $\varepsilon_{i}^{k}$ by means of the equations

$$
\begin{aligned}
& \varepsilon_{i}^{\kappa} \varepsilon_{\kappa}^{h}=A_{i}^{h} \quad \text { (unit affinor) } \\
& \varepsilon_{i}^{\kappa} q_{\kappa}=0 .
\end{aligned}
$$

Multiplication with $\varepsilon_{\lambda}^{i}$ gives

$$
\varepsilon_{\lambda}^{\kappa}=\varepsilon_{i}^{\kappa} \varepsilon_{\lambda}^{i}=\mathscr{A}_{\lambda}^{\kappa}-x^{\kappa} q_{\lambda}
$$

\footnotetext{
1 A. P. D., p. 11.
} 
2. The induced affine connection.

We shall now prove that the quantities

$$
\Gamma_{j i}^{h}=\varepsilon_{\kappa j i}^{h \mu \lambda} \Pi_{\mu \lambda}^{\kappa}-\varepsilon_{j i}^{\mu \lambda} \partial_{\mu} \varepsilon_{\lambda}^{h}, \quad\left(\varepsilon_{\kappa j i}^{h \mu \lambda}=\varepsilon_{\kappa}^{h} \varepsilon_{j}^{\mu} \varepsilon_{i}^{\lambda}\right)
$$

are the coefficients of an affine connection, which gives the same system of paths as the projective connection $\Pi_{\mu \lambda}^{k}$. The system of geodesics, defined by the projective connection $\Pi_{\mu \lambda}^{\kappa}$, is given by the differential equations (2.7). If $x^{\kappa}=x^{\kappa}(t)$ is the equation of a path in homogeneous coordinates, then the non-homogeneous equation is given by

$$
\xi^{h}=\xi^{h}\left(x^{\kappa}(t)\right)=\xi^{h}(t) .
$$

From this equation we have

$$
\begin{aligned}
& \frac{d \xi^{h}}{d t}=\varepsilon_{\lambda}^{h} \frac{d x^{\lambda}}{d t}, \\
& \frac{d^{2} \xi^{h}}{d t^{2}}=\varepsilon_{\lambda}^{h} \frac{d^{2} x^{\lambda}}{d t^{2}}+\frac{d x^{\mu}}{d t} \frac{d x^{\lambda}}{d t} \partial_{\mu} \varepsilon_{\lambda}^{h} .
\end{aligned}
$$

Consequently

$$
\begin{aligned}
\frac{d^{2} \xi^{h}}{d t^{2}}+\Gamma_{j i}^{h} \frac{d \xi^{j}}{d t} \frac{d \xi^{i}}{d t} & =\varepsilon_{\kappa}^{h}\left(\frac{d^{2} x^{\kappa}}{d t^{2}}+\Pi_{\rho \sigma}^{\kappa} \varepsilon_{\mu \lambda}^{\rho \sigma} \frac{d x^{\mu}}{d t} \frac{d x^{\lambda}}{d t}\right) \\
& +\left(\partial_{\mu} \varepsilon_{\lambda}^{h}-\varepsilon_{\mu \lambda}^{\rho \sigma} \partial_{\rho} \varepsilon_{\sigma}^{h}\right) \frac{d x^{\mu}}{d t} \frac{d x^{\lambda}}{d t} .
\end{aligned}
$$

The transvection $\Pi_{\mu \lambda}^{\kappa} x^{\mu}$ is zero by (2.14). Hence

$$
\Pi_{\rho \sigma}^{\kappa} \varepsilon_{\mu \lambda}^{\rho \sigma}=\Pi_{\mu \lambda}^{\kappa} \text {. }
$$

Furthermore we have from (3.6) and the definition of $\varepsilon_{\mu}^{h}$

$$
\varepsilon_{\mu \lambda}^{\rho \sigma} \partial_{\rho} \varepsilon_{\sigma}^{h}=\partial_{\mu} \varepsilon_{\lambda}^{h}+q_{\mu} \varepsilon_{\lambda}^{h}+q_{\lambda} \varepsilon_{\mu}^{h} .
$$

Substituting these expressions in (3.10) we see from (2.7) that the right hand side is proportional to $\frac{d \xi^{h}}{d t}$. Hence the non-homogeneous differential equations for the geodesics are

$$
\frac{d^{2} \xi^{h}}{d t^{2}}+\Gamma_{j i}^{h} \frac{d \xi^{j}}{d t} \frac{d \xi^{i}}{d t}=a \frac{d \xi^{h}}{d t}
$$

These equations show that the $\Gamma_{j i}^{h}$ defined by (3.7) transform as the coefficients of an affine connection. This connection is called the induced affine connection. It defines the same system of paths as the projective connection.

If we choose another projective covariant vectorfield $q_{\lambda}$, then 
(3.7) defines another affine connection, but this connection gives the same system of paths and can, therefore, be obtained from the connection $\Gamma_{j i}^{h}$ by a projective transformation of connection (1.4).

The curvature tensor of the affine connection $\Gamma_{j i}^{h}$ is defined by formula (1.7), namely

$$
R_{\dot{k} j i{ }^{h}}=2 \partial_{[k} \Gamma_{j] i}^{h}+2 \Gamma_{[k|\ell|}^{h} \Gamma_{j] i}^{l} .
$$

When the expressions (3.7) for $\Gamma_{j i}^{h}$ are substituted in the above equations, we find, after some calculation,

$$
R_{\dot{k} j i}^{h}=\varepsilon_{\kappa k j i}^{h \nu \mu \lambda} N_{i j i{ }^{\kappa}}-2 q_{[k j} A_{i}^{h}+2 A_{[k}^{h} q_{j] i},
$$

where

$$
q_{j i}=\varepsilon_{j i}^{\nu \mu} \nabla_{\nu} q_{\mu}
$$

The projector of curvature $N_{\nu \mu \dot{\lambda}}{ }^{*}$ has according to (2.9) and (2.14) the properties

$$
N_{\nu \mu \dot{\lambda}}{ }^{\kappa} x^{\nu}=0, \quad N_{\mu \lambda}=0 .
$$

Contraction of (3.15) with respect to the indices $k$ and $h$ gives therefore

$$
\begin{aligned}
R_{j i} & =-2 q_{[j i]}+n q_{j i}-q_{j i}, \\
R_{(j i)} & =(n-1) q_{(j i)},
\end{aligned}
$$

where the round brackets indicate symmetrization with respect to $i$ and $j$.

3. The parameter $p$ as independent variable.

Let $u^{a}$ be a preferred system of homogeneous parameters on the paths. Then the differential equations for the paths are (2.22)

$$
\partial_{c} B_{b}^{\kappa}+\Pi_{\mu \lambda}^{\kappa} B_{c b}^{\mu \lambda}=0 .
$$

If $x^{\kappa}=x^{\kappa}\left(u^{0}, u^{1}\right)$ is the equation of a path in homogeneous coordinates, then

$$
\xi^{h}=\xi^{h}\left(x^{\kappa}\left(u^{0}, u^{1}\right)\right)=\xi^{h}\left(u^{0}, u^{1}\right)=\xi^{h}\left(1, \frac{u^{1}}{u^{0}}\right)=\xi^{h}(p)
$$

is the equation of the same path in non-homogeneous coordinates with $p$ as independent variable. Differentiation with respect to $u^{1}$ gives

$$
\begin{gathered}
\varepsilon_{\mu}^{h} B_{1}^{\mu}=\left(d_{p} \xi^{h}\right) \frac{1}{u^{0}}, \quad d_{p} \xi^{h}=\frac{d \xi^{h}}{d p}, \\
\left(\partial_{\nu} \varepsilon_{\mu}^{h}\right) B_{11}^{\nu \mu}+\varepsilon_{\mu}^{h} \partial_{1} B_{1}^{\mu}=\left(d_{p}^{2} \xi^{h}\right)\left(\frac{1}{u^{0}}\right)^{2} .
\end{gathered}
$$


Consequently we have

$$
d_{p}^{2} \xi^{h}+\Gamma_{j i}^{h}\left(d_{p} \xi^{j}\right)\left(d_{p} \xi^{i}\right)=\left(u^{0}\right)^{2}\left[\varepsilon_{\mu}^{h} \partial_{1} B_{1}^{\mu}+\left(\partial_{\mu} \varepsilon_{\lambda}^{h}\right) B_{1}^{\mu \lambda}+\Gamma_{j i}^{h} \varepsilon_{\mu \lambda}^{j i} B_{1}^{\mu \lambda}\right] .
$$

From the equation (3.7) it follows by transvection with $\varepsilon_{\mu \lambda}^{j i}$ in consequence of (3.12)

$$
\Gamma_{j i}^{h} \varepsilon_{\mu \lambda}^{j i}=\varepsilon_{\kappa}^{h} \Pi_{\mu \lambda}^{\kappa}-\partial_{\mu} \varepsilon_{\lambda}^{h}-q_{\mu} \varepsilon_{\lambda}^{h}-q_{\lambda} \varepsilon_{\mu}^{h} .
$$

Substituting this expression in (3.24) we get

$$
\begin{aligned}
d_{p}^{2} \xi^{h}+\Gamma_{j i}^{h}\left(d_{p} \xi^{j}\right)\left(d_{p} \xi^{i}\right) & =\left(u^{0}\right)^{2}\left[\varepsilon_{\kappa}^{h}\left(\partial_{1} B_{1}^{\kappa}+\Pi_{\mu \lambda}^{\kappa} B_{11}^{\mu \lambda}\right)-2 q_{\mu} \varepsilon_{\lambda}^{h} B_{11}^{\mu \lambda}\right] \\
& =-2 u^{0} q_{\mu} B_{1}^{\mu} d_{p} \xi^{h}=-2 a d_{p} \xi^{h},
\end{aligned}
$$

according to (3.20). The coefficient $\alpha$ is a function of $p$ and we find by differentiation

$$
\begin{aligned}
d_{p} \alpha & =d_{p}\left(u^{0} q_{\mu} B_{1}^{\mu}\right)=\left(u^{0}\right)^{2} \frac{\partial}{\partial u^{1}}\left(q_{\mu} B_{1}^{\mu}\right) \\
& =\left(u^{0}\right)^{2} B_{11}^{\mu \lambda}\left(\partial_{\mu} q_{\lambda}-\Pi_{\mu \lambda}^{\kappa} q_{\kappa}\right)=\left(u^{0}\right)^{2} B_{11}^{\mu \lambda} \nabla_{\mu} q_{\lambda} .
\end{aligned}
$$

From (3.16) it follows, by multiplication with $\varepsilon_{\lambda \kappa}^{j i}$, that

$$
\varepsilon_{\lambda \kappa}^{j i} q_{j i}=\varepsilon_{\lambda \kappa}^{\nu \mu} \nabla_{\nu} q_{\mu}=\nabla_{\Lambda} q_{\kappa}-x^{\nu} q_{\Lambda} \varepsilon_{\kappa}^{\mu} \nabla_{\nu} q_{\mu}-x^{\mu} q_{\kappa} \nabla_{\lambda} q_{\mu}=\nabla_{\lambda} q_{\kappa}+q_{\lambda} q_{\kappa},
$$

the components $q_{\lambda}$ being homogeneous of degree -1 . Substitution in (3.27) gives

$$
d_{p} a=\left(u^{0}\right)^{2} B_{11}^{\mu \lambda}\left(\varepsilon_{\mu \lambda}^{j i} q_{j i}-q_{\mu} q_{\lambda}\right),
$$

for which we may write according to (3.19), (3.22) and using the definition of $a(3.26)$,

$$
d_{p} a+a^{2}-\frac{1}{n-1} R_{j i}\left(d_{p} \xi^{j}\right)\left(d_{p} \xi^{i}\right)=0 .
$$

These equations together with the differential equations (3.26) of the system of paths are identical with the equations (1.11) and (1.13) if we put $p=\pi$. This means that the projective parameter $p$ defined in $\S 2$ is a projective normal parameter. 